\title{
Mass correction and deformation of slowly rotating anisotropic neutron stars based on Hartle-Thorne formalism
}

\author{
M. L. Pattersons ${ }^{\mathrm{a}} \mathbb{D}$, A. Sulaksono ${ }^{\mathrm{b}}$ \\ Department of Physics, University of Indonesia, Kampus UI Depok, Depok 16424, Indonesia
}

Received: 18 March 2021 / Accepted: 25 July 2021 / Published online: 5 August 2021

(C) The Author(s) 2021

\begin{abstract}
Due to their compactness, neutron stars are the best study matter in high density and strong-field gravity. Hartle and Thorne have proposed a good approximation or perturbation procedure within general relativity for slowly rotating relativistic stars by assuming the matter inside the stars is an ideal isotropic fluid. This study extends the analytical Hartle-Thorne formalism for slowly rotating neutron stars, including the possibility that the neutron star pressure can be anisotropic. We study the impact of neutron stars' anisotropy pressure on mass correction and deformation numerically. For the anisotropic model, we use the Bowers-Liang model. For the equation of state of neutron stars, we use a relativistic mean-field BSP parameter set with the hyperons, and for the crust equation of state, we use the one of Miyatsu et al. We have found that the mass of neutron stars increases but the radius decreases by increasing $\lambda_{B L}$ value. Therefore, the NS compactness increases when $\lambda_{B L}$ becomes larger. This fact leads to a condition in which NS is getting harder to deformed when the $\lambda_{B L}$ increased.
\end{abstract}

\section{Introduction}

It has been understood that the evolution of the massive stars whose mass in the range $8-25 M_{\odot}$ is ended when neutron stars (NSs) formed. The corresponding formation is due to the stars' gravitational collapse during Type-II, Ib, or Ic supernova explosion phenomena [1]. NSs have some convincing observational signatures, such as pulse period, masses (of NS in a binary system), thermal emission, glitches [2]. The corresponding pieces of information through X-rays, $\gamma$-rays, and neutrinos sources can be used to extract the NS properties [1]. NSs are the densest stars. Their density is roughly about $10^{4}$ times denser than that of the earth. Therefore, NSs are

\footnotetext{
${ }^{a}$ e-mail: muhammad.lawrence@ui.ac.id (corresponding author)

be-mail: anto.sulaksono@sci.ui.ac.id
}

the best avenues to study matter at high density and strongfield gravity simultaneously. However, up to now, NS's matter composition and gravity are not entirely understood [3,4]. We need to note that accurate measurements of massive pulsars have been reported in Refs. [5-9]. These measurements provide a maximum mass limit of NS around $2.0 M_{\odot}$. Several constraints of NSs radii from X-ray sources also studied up to the recent years (see Ref. [10] and the references therein for the discussions). The simultaneously mass and radius constraints from NICER using PSR J0030+0451 pulsar [11-13]. The tidal deformability gravitational waves (GW) observations of coalescence NSs with LIGO and VIGO can also provide NS mass and radius constraints [14-16]. It also needs to note that the LIGO/Virgo collaboration recently reported the GW190814 observation of the merger of a black hole (BH) of mass (22.2-24.3) $M_{\odot}$ and a secondary massive compact object with mass $(2.50-2.67) M_{\odot}$ [17]. If the secondary object is NS, then the mass is significantly larger than the known maximum NS mass observed.

The equation of state (EOS) of NS as crucial information input to know the radius and the maximum mass of NS is determined from NS matter composition [18]. Many authors have proposed phenomenological models or the ones based on many-body approaches to calculating EOS. The phenomenological approaches, either relativistic or nonrelativistic, are based on effective interactions developed to reproduce finite nuclei and nuclear matter properties. The Skyrme Hartree-Fock (SHF) and relativistic mean-field (RMF) models belong to this approach. The recent discussions of the application status of RMF models in NS EOS can be consulted to Refs. [19,20]. On the other hand, many-body microscopic models are based on two- and three-body forces that describe scattering data in free space and the properties of the deuteron [1]. To this end, we need to emphasize that NS's properties such as mass, radius, and spin are sensitively influenced by the nature of the NS EOS [21]. Please see the recent review of NS EOS in Refs. [22,23]. 
Due to the strong gravity of NS, one must use general relativity to describe the NS structure. Up to now, it is common that NS is modeled as a static, spherically symmetric, and non-rotating object while the matter is described by isotropic perfect fluid [2]. In this way, one can use TolmanOppenheimer-Volkoff (TOV) equation [24] to calculate NSs structures. The situation becomes relatively more complicated when we assume that NS can rotate and becomes deformed due to the rotation. Hartle [25], and then Hartle and Thorne [26] have proposed a magnificent approximation to handle this problem, i.e., the approximation of slowly rotating relativistic stars. Once one employs the Hartle-Thorne (HT) formalism, deformation and mass correction of the relativistic stars can be calculated. The pioneering work of Hartle and Thorne has been investigated in many studies. According to Ref. [27], HT approach is relative better than those of Manko [28], and Cook-Shapiro-Teukolsky (CST) [29].

In many works, the NS matter is usually modeled as an isotropic fluid. However, it is natural to expect that there is a possibility of the appearance of anisotropic pressure, i.e., the unequal value between radial and tangential pressures inside NS matter. For details, please see Refs. [3,21,30-33] and please also see the references therein. We need to note that the anisotropic pressure might also exist in other kinds of compact stars, such as strange quark stars [34] and please also see the references therein. Many factors can cause anisotropic pressure in compact objects due to matter properties which are linking to each other such as boson condensations, the existence of solid core, different kinds of phase transition, as well as the presence of strong magnetic and electric field (Please see Ref. [30] and the references therein.), and or due to the impact of modified geometry [35-39]. Studies on static anisotropic NSs to obtain their mass correction and deformation have been studied; for example, it is discussed in Refs. [21,31,32,40]. In Refs. [21,31,32], the perturbation in metric appears due to NS's magnetic field is modeled as the source of anisotropic pressure of NS. Note that the formalism is ana$\log$ to that used in perturbation matric due to HT formalism for rotation. However, the impact of rotation is still neglected in their works. It means that their works are not too realistic since celestial objects are normally rotating due to the law of angular momentum conservation [41]. It is worthed noting that many authors have investigated the numerical calculation of rotating NSs by considering NSs as stars with isotropic pressure within HT formalism. Please see Ref. [2] as an example. However, it is evident from many studies that the anisotropic pressure is more realistic to describe NSs. Please see Refs. [3,21,30-33] and the references therein.

Considering these factors and the fact that the HT model is better than the Manko and CST model, we argue that it is essential to extend HT formalism by including anisotropic pressure. Therefore, we can approach perturbations attributed to NS as a complete form that contains both anisotropic pressure and rotation. In this study, we reformulated the formalism of mass correction and deformation of slowly rotating anisotropic NS based on HT formalism, so we still used HT metric and followed Einstein tensor calculations done by Hartle and Thorne [25,26]. We added anisotropic pressure in the energy-momentum tensor and followed by using Hartle and Thorne's procedure to calculate Einstein field equations. We then applied the result to calculate the mass correction and deformation of NS. We have obtained the results by employing numerical calculations. Here, we use the Bowers-Liang (BL) model for the anisotropic pressure of NS as a numerical case study [42]. The EOS of the core of NS is calculated using the RMF model with the BSP parameter set $[43,44]$ under which the standard SU(6) prescription and hyperon potential depths [45] are utilized to determine the hyperon coupling constants. For the inner and outer crusts, we use the crust EOS obtained from Miyatsu et al. [46].

The structure of this paper is, in Sect. 2, we discuss the formalism used. Then, in Sect. 3, we discuss the obtained numerical results, concluding in Sect. 4.

\section{Formalism}

To make the discussion self-contained, in Sect. 2.1, we briefly review the standard HT analytical formalism for isotropic stars, while the the one for anisotropic is given in Sect. 2.2.

2.1 HT formalism for mass correction and deformation of slowly rotating relativistic stars

Here we review briefly the HT formalism for isotropic stars. In this paper we use geometrized units $G=c=1$. The HT metric can be expressed as [2]

$$
\begin{aligned}
d s^{2}= & -e^{2 v} d t^{2}+e^{2 \lambda} d r^{2}+r^{2} e^{2 \psi}(d \phi-\omega d t)^{2} \\
& +r^{2} e^{2 \mu} d \theta^{2}
\end{aligned}
$$

where $\omega$ denotes the angular velocity of local inertial frame, and it is proportional to the angular velocity of the star $\Omega$.

The exponential factors in each terms of the right-hand side of Eq. (1) are expanded as

$$
\begin{aligned}
e^{2 v} & =e^{2 \varphi}\left[1+2\left(h_{0}+h_{2} P_{2}(\cos \theta)\right)\right], \\
e^{2 \lambda} & =\left[1+\frac{2}{r}\left(m_{0}+m_{2} P_{2}(\cos \theta)\right)\left(1-\frac{2 m(r)}{r}\right)^{-1}\right], \\
e^{2 \psi} & =\sin ^{2} \theta\left[1+2\left(v_{2}-h_{2}\right) P_{2}(\cos \theta)\right], \\
e^{2 \mu} & =\left[1+2\left(v_{2}-h_{2}\right) P_{2}(\cos \theta)\right],
\end{aligned}
$$


where $h_{0}, h_{2}, m_{0}, m_{2}$, and $v_{2}$ are perturbation functions; $P_{2}(\cos \theta)$ is the second order of Legendre polynomial; $e^{2 \varphi}$ and $m(r)$ are functions which are bound by constraints [25]

$\frac{d \varphi}{d r}=\frac{m(r)+4 \pi r^{3} p(r)}{r(r-2 m(r))}$,

and

$\frac{d m}{d r}=4 \pi r^{2} \epsilon(p)$.

where $\epsilon$ denotes energy density, $p$ denotes pressure of the star. TOV equation [24] can be obtained from Bianchi identity and Eq. (6). It is written as

$\frac{d p}{d r}=-\frac{(\epsilon+p)\left(m(r)+4 \pi r^{3} p(r)\right)}{r(r-2 m(r))}$.

In order to obtain the perturbation functions $h_{0}, h_{2}, m_{0}$, and $m_{2}$, one has to solve the Einstein equations as

$R_{v}^{\mu}-\frac{1}{2} \delta_{v}^{\mu} R=8 \pi T_{\nu}^{\mu}$

By calculating $(t, t)$ and $(r, r)$ components of Einstein equations, one can obtain

$$
\begin{aligned}
\frac{d m_{0}}{d r}= & 4 \pi r^{2} \frac{d \epsilon}{d p}(\epsilon+p) p_{0}^{*}+\frac{1}{12} j^{2} r^{4}\left(\frac{d \bar{\omega}}{d r}\right)^{2} \\
& -\frac{1}{3} r^{3} \frac{d j^{2}}{d r} \bar{\omega}^{2}, \\
\frac{d p_{0}^{*}}{d r}= & -\frac{m_{0}\left(1+8 \pi r^{2} p\right)}{(r-2 m)^{2}}-\frac{4 \pi(\epsilon+p) r^{2}}{(r-2 m)} p_{0}^{*} \\
& +\frac{1}{12} \frac{r^{4} j^{2}}{(r-2 m)}\left(\frac{d \bar{\omega}}{d r}\right)^{2}+\frac{1}{3} \frac{d}{d r}\left(\frac{r^{3} j^{2} \bar{\omega}^{2}}{r-2 m}\right),
\end{aligned}
$$

where $p_{0}^{*}=-h_{0}+\frac{1}{3} r^{2} e^{-2 \varphi} \bar{\omega}+h$, and $h$ is a constant that is needed so that $h_{0}$ is continuous across the surface of the star; $\bar{\omega}=\Omega-\omega$, i.e. angular velocity of the star relative to the local inertial frame; and $j=e^{-\varphi}\left(1-\frac{2 m(r)}{r}\right)^{1 / 2}$. Boundary conditions for both $m_{0}$ and $p_{0}^{*}$ are zero at $r=0$ since they vanish at the origin.

A limit for the rotation of a star is called Kepler frequency $\Omega_{K}$. It can be approximated as [4]

$\Omega_{K} \approx\left[1+\frac{\omega(R)}{\Omega_{K}}-2\left(\frac{\omega(R)}{\Omega_{K}}\right)^{2}\right]^{-1 / 2} \sqrt{\frac{M}{R^{3}}}$.

where $M$ is mass of the star, and $R$ is radius of the star. Further,

$\frac{\omega(R)}{\Omega_{K}}=\frac{2 I}{R^{3}}$, where $I$ is moment of inertia. Based on Ref. [3], $I$ can be approximated as

$I \approx 0.24 M R^{2}$.

Considering Eqs. (12) and (14), we define $\Omega$ which is always less than Kepler frequency in slow rotating approximation, i.e.

$\Omega \lesssim\left[1+0.48 \frac{M}{R}-2\left(0.24 \frac{M}{R}\right)^{2}\right]^{-1 / 2} \sqrt{\frac{M}{R^{3}}}$.

In this calculation concatenation, one has to find $\bar{\omega}$ by solving a differential equation, i.e.

$\frac{1}{r^{4}} \frac{d}{d r}\left(r^{4} j \frac{d \bar{\omega}}{d r}\right)+\frac{4}{r} \frac{d j}{d r} \bar{\omega}=0$.

Boundary conditions at $r=0$ that satisfies Eq. (16) are $\bar{\omega}=$ $\omega_{c}$ and $\frac{d \bar{\omega}}{d r}=0$, where $\omega_{c}$ can be chosen arbitrarily.

The mass correction can be written as

$\delta M=m_{0}(R)+\frac{J^{2}}{R^{3}}$,

where $R$ is the radius of the star without deformation, and $J$ denotes angular momentum of the star.

From the $(\theta, \theta),(\phi, \phi),(\theta, r)$, and $(r, r)$ components of Einstein equations, one can obtains

$$
\begin{aligned}
\frac{d v_{2}}{d r}= & -2 \frac{d \varphi}{d r} h_{2}+\left(\frac{1}{r}+\frac{d \varphi}{d r}\right) \\
& \times\left[-\frac{1}{3} r^{3} \frac{d j^{2}}{d r} \bar{\omega}^{2}+\frac{1}{6} j^{2} r^{4}\left(\frac{d \bar{\omega}}{d r}\right)^{2}\right], \\
\frac{d h_{2}}{d r}= & -2 \frac{d \varphi}{d r} h_{2}+\frac{r}{r-2 m}\left(2 \frac{d \varphi}{d r}\right)^{-1} \\
& \times\left\{8 \pi(\epsilon+p)-\frac{4 m}{r^{3}}\right\} h_{2}-\frac{4 v_{2}}{r(r-2 m)} \\
& \times\left(2 \frac{d \varphi}{d r}\right)^{-1}+\frac{1}{6}\left[\frac{d \varphi}{d r} r-\frac{1}{r-2 m}\left(2 \frac{d \varphi}{d r}\right)^{-1}\right] \\
& \times r^{3} j^{2}\left(\frac{d \bar{\omega}}{d r}\right)^{2}-\frac{1}{3}\left[\frac{d \varphi}{d r} r+\frac{1}{r-2 m}\left(2 \frac{d \varphi}{d r}\right)^{-1}\right] \\
& \times r^{2} \frac{d j^{2}}{d r} \bar{\omega}^{2} .
\end{aligned}
$$

with the boundary conditions

$v_{2}(0)=h_{2}(0)=v_{2}(\infty)=h_{2}(\infty)=0$.

There is a formula $[2,25]$

$p_{2}^{*}=-h_{2}-\frac{1}{3} r^{2} e^{-2 \varphi} \bar{\omega}^{2}$. 
The deformation is expressed as

$\delta r=\xi_{0}(R)+\xi_{2}(R) P_{2}(\cos \theta)$,

where

$$
\begin{aligned}
& \xi_{0}(r)=-p_{0}^{*}(\epsilon+p)\left(\frac{d p}{d r}\right)^{-1}, \\
& \xi_{2}(r)=-p_{2}^{*}(\epsilon+p)\left(\frac{d p}{d r}\right)^{-1} .
\end{aligned}
$$

From this point, we can calculate the radius of the pole $R_{p}$ and the radius of the equator $R_{e}$,

$R_{p}=R+\xi_{0}+\xi_{2}$,

$R_{e}=R+\xi_{0}-\frac{1}{2} \xi_{2}$.

We can also find the eccentricity of the star, i.e.

$e=\sqrt{1-\frac{R_{p}}{R_{e}}}$

The star is spherically symmetric if the value of $e$ is equal to 0 , and its shape is getting more oblate as the value of $e$ becomes closer to one.

\subsection{Modified HT formalism due to anisotropic pressure of NS}

Here we present the analytical results of the impact of anisotropic pressure correction on HT formalism. The energy-momentum tensor of anisotropic NS [30,33] can be written as

$T_{v}^{\mu}=\epsilon u^{\mu} u_{v}+q \delta_{v}^{\mu}+\sigma k^{\mu} k_{v}$,

where $\sigma=p-q$, and $k_{v}$ is a unit radial vector that satisfies $u^{\mu} k_{v}=0$.

In BL model, $\sigma$ is expressed as $[3,42]$

$\sigma=-\lambda_{B L} \frac{G \epsilon r^{2}}{3}\left(1+\frac{3 p}{\epsilon}\right) \frac{\left(1+\frac{p}{\epsilon}\right)}{1-\frac{2 G m(r)}{r}}$,

where $\lambda_{B L}$ is defined as the strength of anisotropic terms of the model, and $G$ is the universal constant of gravity.

TOV equation for anisotropic NS [30] is expressed by

$\frac{d p}{d r}=-\frac{(\epsilon+p)\left(m(r)+4 \pi r^{3} p(r)\right)}{r(r-2 m(r))}-\frac{2 \sigma}{r}$.

By reformulating HT model which can be compatible for slowly rotating anisotropic NS, several eqs. are modified. We followed calculation procedure for Einstein equations. which is written in Ref. [25]. The multipole expansion terms are limited to $l=2$. One can obtain the expansion terms of Einstein tensor and Ricci tensor. Those are written as

$$
\begin{aligned}
& \left(\delta G_{t}^{t}\right)_{l=0}=\frac{j}{6 r^{2}}\left[8 r^{3} \frac{d j}{d r} \omega(\Omega-\omega)+j r^{4}\left(\frac{d \omega}{d r}\right)^{2}\right] \\
& -\frac{d m_{0}}{d r} \frac{2}{r^{2}} \\
& \left(\delta G_{t}^{t}\right)_{l=2}=\frac{j}{6 r^{2}}\left[8 r^{3} \frac{d j}{d r} \omega(\Omega-\omega)+j r^{4}\left(\frac{d \omega}{d r}\right)^{2}\right] \\
& -\frac{d m_{2}}{d r} \frac{2}{r^{2}}+\left(1-\frac{2 m}{r}\right) \\
& \times\left[2\left(\frac{d^{2} v_{2}}{d r^{2}}-\frac{d^{2} h_{2}}{d r^{2}}\right)+\frac{6}{r}\left(\frac{d v_{2}}{d r}-\frac{d h_{2}}{d r}\right)\right] \\
& -2\left(\frac{d v_{2}}{d r}-\frac{d h_{2}}{d r}\right) \frac{d}{d r}\left(\frac{m}{r}\right) \\
& -\frac{6 m_{2}}{r^{2}(r-2 m)}-\frac{4\left(v_{2}-h_{2}\right)}{r^{2}} \text {, } \\
& \left(\delta G_{r}^{r}\right)_{l=0}=\frac{1}{6} r^{2} j^{2}\left(\frac{d \omega}{d r}\right)^{2}-\frac{2 m_{0}}{r^{2}}\left(2 \frac{d \varphi}{d r}+\frac{1}{r}\right) \\
& +\left(1-\frac{2 m}{r}\right) \frac{2}{r} \frac{d h_{0}}{d r}, \\
& \left(\delta G_{r}^{r}\right)_{l=2}=-\frac{1}{6} r^{2} j^{2}\left(\frac{d \omega}{d r}\right)^{2}-\frac{2 m_{2}}{r^{2}}\left(2 \frac{d \varphi}{d r}+\frac{1}{r}\right) \\
& +\left(1-\frac{2 m}{r}\right) \frac{2}{r} \frac{d h_{2}}{d r}-\frac{6 h_{2}}{r^{2}} \\
& +\left(1-\frac{2 m}{r}\right)\left(\frac{d v_{2}}{d r}-\frac{d h_{2}}{d r}\right)\left(2 \frac{d \varphi}{d r}+\frac{2}{r}\right) \\
& -\frac{4\left(v_{2}-h_{2}\right)}{r} \text {, } \\
& \left(\delta R_{r \theta}\right)_{l=0}=0 \\
& \left(\delta R_{r \theta}\right)_{l=2}=h_{2}\left(\frac{1}{r}-\frac{d \varphi}{d r}\right)-\frac{d v_{2}}{d r}+\frac{m_{2}}{r-2 m} \\
& \times\left(\frac{1}{r}+\frac{d \varphi}{d r}\right),
\end{aligned}
$$

$$
\begin{aligned}
\left(R_{\theta}^{\theta}-R_{\phi}^{\phi}\right)= & \frac{1}{2} \sin ^{2} \theta\left[-\frac{3\left(h_{2}+\frac{m_{2}}{r-2 m}\right)}{r^{2}}\right] \\
& +\frac{j^{2} r^{2}}{4}\left(\sin ^{2} \theta \frac{d \omega}{d r}\right)^{2}+\frac{1}{2} r \omega(\Omega-\omega) \\
& \times\left(\frac{d j^{2}}{d r}\right) \sin ^{2} \theta .
\end{aligned}
$$

According to Ref. [25], correction of Einstein tensor can be written as

$\Delta G_{\mu}^{v}=\delta G_{\mu}^{v}+\xi \frac{\partial}{\partial r}\left[G_{\mu}^{v(0)}\right]$ 
where $G_{\mu}^{v(0)}$ is unperturbed Einstein tensor. Recall unperturbed Einstein equations $G_{\mu}^{\nu(0)}=8 \pi T_{\mu}^{\nu(0)}$, where $T^{\nu(0)}$ is energy-momentum tensor which is not perturbed by rotation, so one can obtain

$\Delta G_{\mu}^{\nu}=\delta G_{\mu}^{\nu}+8 \pi \xi \frac{\partial}{\partial r}\left[T_{\mu}^{\nu(0)}\right]$.

The explicit forms of correction of Einstein tensor are written as

$$
\begin{aligned}
\Delta G_{t}^{t} & =\delta G_{t}^{t}-8 \pi \xi \frac{\partial \epsilon}{\partial r}, \\
\Delta G_{r}^{r} & =\delta G_{r}^{r}+8 \pi \xi \frac{\partial p}{\partial r}, \\
\Delta G_{\theta}^{\theta} & =\delta G_{\theta}^{\theta}+8 \pi \xi \frac{\partial q}{\partial r}, \\
\Delta G_{\phi}^{\phi} & =\delta G_{\phi}^{\phi}+8 \pi \xi \frac{\partial q}{\partial r}, \\
\Delta G_{r}^{\theta} & =\delta G_{r}^{\theta} .
\end{aligned}
$$

The mathematical entity $\xi$ is expanded as

$\xi=\xi_{0}(r)+\xi_{2}(r) P_{2}(\cos \theta)+\cdots$.

Here we limited the expansion terms of $\xi$ to $l=2$.

Considering Eq. (28), so we have new forms of $p_{0}^{*}$ and $p_{2}^{*}$, i.e.

$p_{0}^{*}=-\xi_{0}\left(\frac{1}{\epsilon+q} \frac{d q}{d r}\right)$,

$p_{2}^{*}=-\xi_{2}\left(\frac{1}{\epsilon+q} \frac{d q}{d r}\right)$,

and $\xi_{0}$ and $\xi_{2}$ can be expressed as

$\xi_{0}=-p_{0}^{*}(\epsilon+p)\left(1-\frac{\sigma}{\epsilon+p}\right)\left(\frac{d p}{d r}-\frac{d \sigma}{d r}\right)^{-1}$,
$\xi_{2}=-p_{2}^{*}(\epsilon+p)\left(1-\frac{\sigma}{\epsilon+p}\right)\left(\frac{d p}{d r}-\frac{d \sigma}{d r}\right)^{-1}$.

Equations (48) and (49) are anisotropic modified forms of Eqs. (23) and (24).

While the correction of energy-momentum tensor for anisotropic NS can be written as

$$
\begin{aligned}
\Delta T_{t}^{t} & =\delta T_{t}^{t}+\xi \frac{\partial \epsilon}{\partial r}, \\
\Delta T_{r}^{r} & =\delta T_{r}^{r}+\xi \frac{\partial p}{\partial r}, \\
\Delta T_{\theta}^{\theta} & =\delta T_{\theta}^{\theta}+\xi \frac{\partial q}{\partial r}, \\
\Delta T_{\phi}^{\phi} & =\delta T_{\phi}^{\phi}+\xi \frac{\partial q}{\partial r},
\end{aligned}
$$

where

$\delta T_{v}^{\mu}=(\Delta \epsilon+\Delta q) u^{\mu} u_{v}+\Delta q \delta_{v}^{\mu}+\Delta \sigma k^{\mu} k_{v}$,

$$
\begin{aligned}
& \Delta \epsilon=(\epsilon+q)\left(\frac{\partial \epsilon}{\partial q}\right) p^{*}=-\frac{\partial \epsilon}{\partial r} \xi, \\
& \Delta q=(\epsilon+q) p^{*}=-\frac{\partial q}{\partial r} \xi, \\
& \Delta \sigma=(\epsilon+q)\left(\frac{\partial \sigma}{\partial q}\right) p^{*}=-\frac{\partial \sigma}{\partial r} \xi .
\end{aligned}
$$

It is easy to show that

$$
\begin{aligned}
& \left(\Delta T_{t}^{t}\right)_{l=0}=-\frac{2}{3}(\epsilon+p) e^{-v} r^{2} \bar{\omega}^{2}\left(1-\frac{\sigma}{\epsilon+p}\right), \\
& \left(\Delta T_{r}^{r}\right)_{l=0}=0 .
\end{aligned}
$$

We step further to Einstein equations for the corrections of the tensors

$\Delta G_{v}^{\mu}=8 \pi \Delta T_{v}^{\mu}$.

With a little algebra, for $\mu=v=t$, and $l=0$, one can obtain

$$
\begin{aligned}
\frac{d m_{0}}{d r}= & 4 \pi r^{2} \frac{d \epsilon}{d p}\left(1-\frac{d \sigma}{d p}\right)^{-1}(\epsilon+p)\left(1-\frac{\sigma}{\epsilon+p}\right) p_{0}^{*} \\
& +\frac{1}{12} j^{2} r^{4}\left(\frac{d \bar{\omega}}{d r}\right)^{2}-\frac{1}{3} r^{3}\left(\frac{d j^{2}}{d r}\right) \bar{\omega}^{2} \\
& \times\left(1-\frac{\sigma}{\epsilon+p}\right),
\end{aligned}
$$

and for $\mu=v=r$, with $l=0$, one can obtain

$$
\begin{aligned}
\frac{d p_{0}^{*}}{d r}= & -\frac{m_{0}\left(1+8 \pi r^{2} p\right)}{(1-2 m)^{2}}-\frac{4 \pi r^{2}(\epsilon+p)}{r-2 m} \\
& \times\left(1-\frac{\sigma}{\epsilon+p}\right) p_{0}^{*}+\frac{1}{12} \frac{r^{4} j^{2}}{r-2 m}\left(\frac{d \bar{\omega}}{d r}\right)^{2} \\
& +\frac{1}{3} \frac{d}{d r}\left(\frac{r^{3} j^{2} \bar{\omega}^{2}}{r-2 m}\right) .
\end{aligned}
$$

Equations (61) and (62) are modified forms of Eqs. (10) and (11), respectively. It is important to keep in mind that both $m_{0}$ and $p_{0}^{*}$ vanish at the origin.

From $(\theta, \theta)$ and $(\phi, \phi)$ components, we can obtain

$T_{\theta}^{\theta}-T_{\phi}^{\phi}=-(\epsilon+p) e^{-v} \bar{\omega}^{2} r^{2} \sin ^{2} \theta\left(1-\frac{\sigma}{\epsilon+p}\right)$.

Recall the Einstein equations for $(\theta, \theta)$ and $(\phi, \phi)$ components

$8 \pi\left(T_{\theta}^{\theta}-T_{\phi}^{\phi}\right)=R_{\theta}^{\theta}-R_{\phi}^{\phi}$.

From Eq. (64), one can obtain

$$
\begin{aligned}
m_{2}= & {\left[-h_{2}-\left(\frac{1}{3}-\frac{2 \sigma}{\epsilon+p}\right) r^{3}\left(j^{2}\right)_{r} \bar{\omega}^{2}+\frac{1}{6} j^{2} r^{4}\left(\bar{\omega}_{r}\right)^{2}\right] } \\
& \times r\left(1-\frac{2 m}{r}\right) .
\end{aligned}
$$


It is clear that non-diagonal components of energy-momentum tensor is equal to zero, so

$\left(\delta T_{r}^{\theta}\right)_{l=2}=\left(\delta T_{r \theta}\right)_{l=2}=0$.

Recall the Einstein equation for $(r, \theta)$ component with $l=2$ $8 \pi\left(\delta T_{r \theta}\right)_{l=2}=\left(\delta R_{r \theta}\right)_{l=2}$,

$$
\begin{aligned}
0= & -\frac{d h_{2}}{d r}+h_{2}\left(\frac{1}{r}-\frac{d \varphi}{d r}\right) \\
& -\left(\frac{d v_{2}}{d r}-\frac{d h_{2}}{d r}\right)+\frac{m_{2}}{r-2 m} \\
& \times\left(\frac{1}{r}+\frac{d \varphi}{d r}\right) \\
\frac{d v_{2}}{d r}= & \frac{m_{2}}{r-2 m}\left(\frac{1}{r}+\frac{d \varphi}{d r}\right)+h_{2} \\
& \times\left(\frac{1}{r}-\frac{d \varphi}{d r}\right) .
\end{aligned}
$$

By inserting Eq. (65) into Eq. (67), we can obtain

$$
\begin{aligned}
\frac{d v_{2}}{d r}= & -2 \frac{d \varphi}{d r} h_{2}+\left(\frac{1}{r}+\frac{d \varphi}{d r}\right) \\
& \times\left[-\left(\frac{1}{3}-\frac{2 \sigma}{\epsilon+p}\right) r^{3} \frac{d j^{2}}{d r} \bar{\omega}^{2}\right] \\
& +\left(\frac{1}{r}+\frac{d \varphi}{d r}\right)\left[\frac{1}{6} j^{2} r^{4}\left(\frac{d \bar{\omega}}{d r}\right)^{2}\right] .
\end{aligned}
$$

Equation (68) is modified form of Eq. (18).

From Eq. (51) it is clear that

$$
\left(\Delta T_{r}^{r}\right)_{l=2}=0 .
$$

Recall the Einstein equation for $(r, r)$ component, with $l=2$

$$
\begin{aligned}
8 \pi\left(\Delta T_{r}^{r}\right)_{l=2}= & \left(\Delta G_{r}^{r}\right)_{l=2}, \\
0= & -\frac{1}{6} r^{2} j^{2}\left(\frac{d \bar{\omega}}{d r}\right)^{2}-\frac{2 m_{2}}{r^{2}}\left(2 \frac{d \varphi}{d r}+\frac{1}{r}\right) \\
& +\left(1-\frac{2 m}{r}\right) \frac{2}{r} \frac{d h_{2}}{d r}-\frac{6 h_{2}}{r^{2}} \\
& +\left(1-\frac{2 m}{r}\right)\left(\frac{d v_{2}}{d r}-\frac{d h_{2}}{d r}\right) \\
& \times\left(2 \frac{d \varphi}{d r}+\frac{2}{r}\right)-\frac{4\left(v_{2}-h_{2}\right)}{r} \\
& +8 \pi \xi \xi_{2} \frac{d p}{d r}, \\
0= & -\frac{1}{6} r^{2} j^{2}\left(\frac{d \bar{\omega}}{d r}\right)^{2}-\frac{2 m_{2}}{r^{2}}\left(2 \frac{d \varphi}{d r}+\frac{1}{r}\right) \\
& +\left(1-\frac{2 m}{r}\right) \frac{2}{r} \frac{d h_{2}}{d r}-\frac{6 h_{2}}{r^{2}} \\
& +\left(1-\frac{2 m}{r}\right)\left(\frac{d v_{2}}{d r}-\frac{d h_{2}}{d r}\right)
\end{aligned}
$$

$$
\begin{aligned}
& \times\left(2 \frac{d \varphi}{d r}+\frac{2}{r}\right)-\frac{4\left(v_{2}-h_{2}\right)}{r} \\
& -8 \pi(\epsilon+p)\left(1-\frac{\sigma}{\epsilon+p}\right) \\
& \times\left(1-\frac{d \sigma}{d p}\right) p_{2}^{*} .
\end{aligned}
$$

Recall the formula [25] $p_{2}^{*}=-h_{2}-\frac{1}{3} e^{-v} r^{2} \bar{\omega}^{2}$, so we can write Eq. (70) as

$$
\begin{aligned}
\frac{d h_{2}}{d r}= & -2 h_{2} \frac{d \varphi}{d r}+\frac{r}{r-2 m}\left(2 \frac{d \varphi}{d r}\right)^{-1} h_{2} \\
& \times 8 \pi(\epsilon+p)\left(1-\frac{\sigma}{\epsilon+p}\right)\left(1-\frac{d \sigma}{d p}\right)^{-1} \\
& -\frac{r}{r-2 m}\left(2 \frac{d \varphi}{d r}\right)^{-1} \frac{4 m}{r^{3}} h_{2} \\
& -\frac{4 v_{2}}{r(r-2 m)}\left(2 \frac{d \varphi}{d r}\right)^{-1}+r^{3} j^{2}\left(\frac{d \bar{\omega}}{d r}\right)^{2} \\
& \times \frac{1}{6}\left[\frac{d \varphi}{d r} r-\frac{1}{r-2 m}\left(2 \frac{d \varphi}{d r}\right)^{-1}\right] \\
& -\frac{1}{3}\left(1-\frac{6 \sigma}{\epsilon+p}\right) \frac{d \varphi}{d r} r^{3}\left(\frac{d j^{2}}{d r}\right) \bar{\omega}^{2} \\
& +\frac{r^{2}}{3}\left(\frac{d j^{2}}{d r}\right) \bar{\omega}^{2} \frac{1}{r-2 m}\left(2 \frac{d \varphi}{d r}\right)^{-1} \\
& \times\left(1-\frac{\sigma}{\epsilon+p}\right)\left(1-\frac{d \sigma}{d p}\right)
\end{aligned}
$$

Equation (71) is modified form of Eq. (71). Boundary conditions for both $v_{2}$ and $h_{2}$ are not changed, i.e. Eq. (20).

For anisotropic NS, The momen of inertia equation in Eq. (16) is modified to be following expression

$\frac{1}{r^{4}} \frac{d}{d r}\left(r^{4} j \frac{d \bar{\omega}}{d r}\right)+\frac{4}{r} \frac{d j}{d r}\left(1-\frac{\sigma}{\epsilon+p}\right) \bar{\omega}=0$.

These modified differential equations due to anisotropic pressure of $h_{0}, h_{2}, m_{0}, m_{2}$, and $v_{2}$ and the one of inertia moment are the main analytical results of this work.

\section{Numerical results}

In numerical calculations, we used Runge-Kutta fourthorder method to solve every differential equation used in this work. For the EOS, we used the model from Ref. [44], and the hyperons are taken into account. The same EOS has been used in several studies, e.g. [3,31,32]. The main differential equations in this work are Eqs. (61), (62), (71), and (67), with boundary conditions $m_{0}(0)=0, p_{0}^{*}(0)=0, v_{2}(0)=0$, and $h_{2}(0)=0$. The values of $m_{0}(R), p_{0}^{*}(R), v_{2}(R)$, and $h_{2}(R)$ are obtained when the Runge-Kutta calculations of differential equations reach $r=R$ due to $P(R)=0$ stop criteria. The 


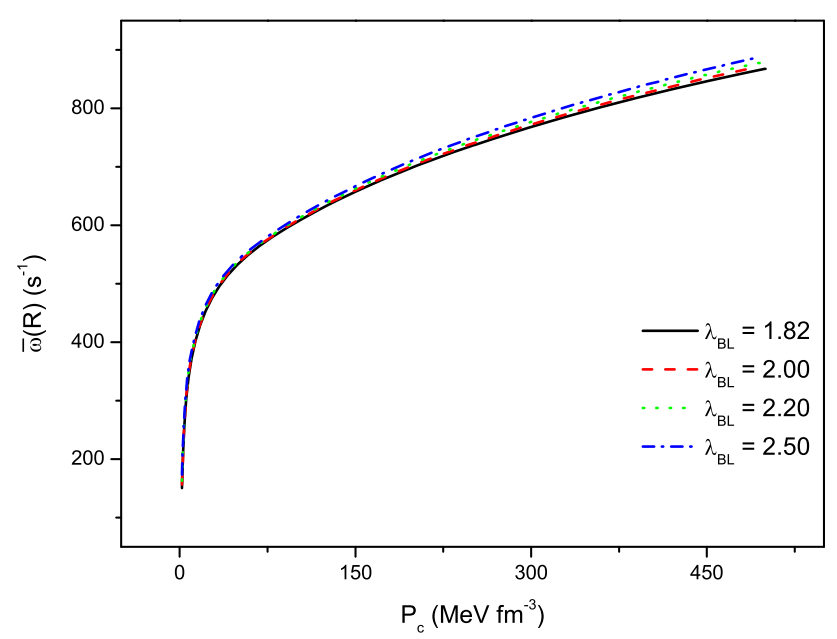

Fig. 1 The center pressure and angular velocity of NS relation. The angular velocity is evaluated at the star's surface, i.e., $\bar{\omega}(R)$. We used four values of $\lambda_{B L}$, which represent the strength of anisotropic terms. The influence of the star's anisotropic pressure can be seen obviously after $P_{c}$ has already exceeded $150 \mathrm{MeV} \mathrm{fm}^{-3}$

value of $m_{0}(R)$ is used to find mass correction so that we can substitute this value to Eq. (17), while the value of $p_{0}^{*}(R)$ is used to find $\xi_{0}(R)$ which is related to the star's deformation. Note that differential equations of $p_{0}^{*}$ and $m_{0}$ are coupled to each other, so we have to solve both simultaneously. The value of $h_{2}(R)$ is needed to find $p_{2}^{*}(R)$ so that we can find $\xi_{2}(R)$ which is related to the star's shape, see Eqs. (21) and (49). The function $v_{2}(r)$ is needed to calculate to help solving $h_{2}$, because $h_{2}$ and $v_{2}$ are coupled to each other (Please see Eqs. (67) and (71).). In order to solve those differential equations, there are components within those equations that have to be obtained from other differential equations, i.e. $p(r)$, $\varphi(r), m(r)$, and $\bar{\omega}(r)$. The solutions are obtained by solving Eqs. (30), (6), (7, respectively), and (72), respectively.

In solving differential equation of angular velocity, we used $\bar{\omega}_{c}=0$ as the boundary condition for $\bar{\omega}$. Note that this value is much smaller than the value used in Ref. [2], i.e. $\bar{\omega}=3000 \mathrm{~s}^{-1}$. The $\bar{\omega}$ initial value used in this work is more realistic for slowly rotating conditions because if we consider that $\bar{\omega}(r)$ increases along with the increment of $r$. Then, by setting relative large value i.e., $\bar{\omega}_{c}=3000 \mathrm{~s}^{-1}$ is rather risky. We could lose the physical meaning that HT formalism is an approximation of slowly rotating relativistic stars. We can see in Fig. 1 that $\bar{\omega}(R)$, i.e., the angular velocities relative to local inertial frame at the star's surface, significantly increase along with the increment of pressure at the center, but it only happens in a range where the values of pressure at the center are relatively small, i.e., between 0 and $150 \mathrm{MeV} \mathrm{fm}^{-3}$. In this range, anisotropic pressure does not influence the relation between the pressure at the center and $\bar{\omega}(R)$ because the curves coincide. Therefore, we cannot observe the significant difference in $\bar{\omega}(R)$ when we employ different values of $\lambda_{B L}$.

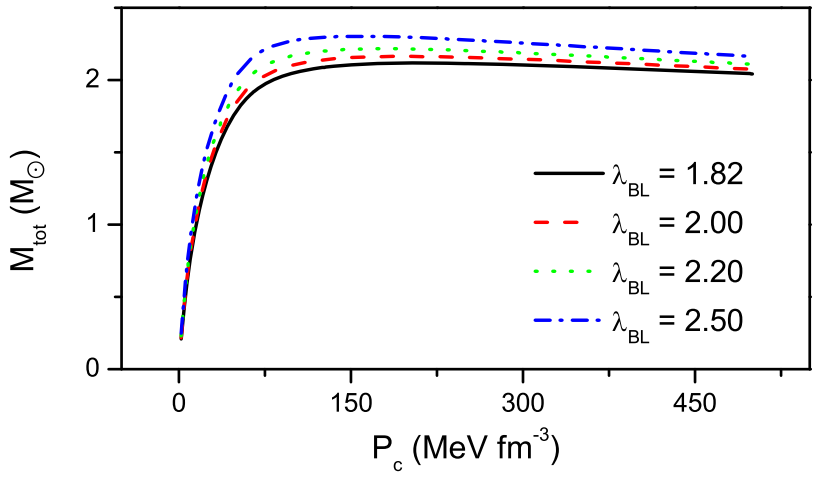

(a)

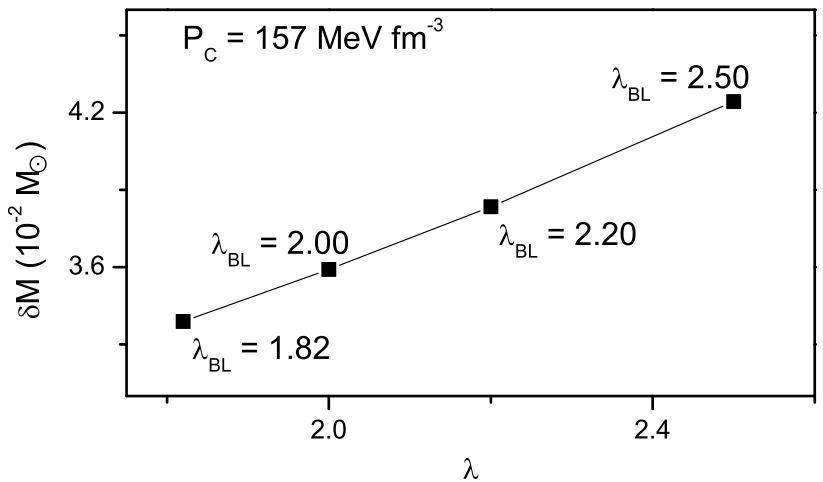

(b)

Fig. 2 Impact of anisotropic pressure on total mass and mass correction of NS. The $\lambda$ are specified in the figures. a The relation between the pressure at the center of the star and total mass. Maximum mass of NS with $\lambda_{B L}=2.50$ (blue dot-dash curve) exceeds $2.3 M_{\odot}$. b Mass correction, i.e. $\delta M$ for different values of $\lambda_{B L}$ at $P_{c}=157 \mathrm{MeV} \mathrm{fm}^{-3}$, which is the point where star with $\lambda_{B L}=2.50$ reach the higher value of mass correction

The influence of the anisotropic pressure on $\bar{\omega}(R)$ can be seen more obviously for the relatively large center pressure value. It occurs when the curves exceed $P_{c}=150 \mathrm{MeV} \mathrm{fm}^{-3}$. We also see that the stars with larger $\lambda_{B L}$ values rotate faster from the curves. Note that according to Eq. (72), $\bar{\omega}$ do not only depend on $\sigma$ as the correction element of anisotropic pressure, it is also dependent on $r$ and other parameters such as pressure and energy density. Note that the fastest angular velocity obtained in this work does not exceed $1000 \mathrm{~s}^{-1}$, which is even much less than the value of $\bar{\omega}_{c}$ used in Ref. [2]. This value indicates that our parameter for $\bar{\omega}_{c}$ is quite safe for describing slow rotating NS.

Figure 2 describes the impact of anisotropic pressure on total mass and NS's mass correction. Total mass is the summation of the mass of a static star that is obtained from Eq. (7) and mass correction, which is obtained from Eq. (17). Fig. 2a shows the relation between the pressure at the center and the star's total mass. We plotted curves for four different values of $\lambda_{B L}$. We can see that total mass increases for every larger value of $\lambda_{B L}$. As $\lambda_{B L}$ decreases to 1.82 , the total mass 


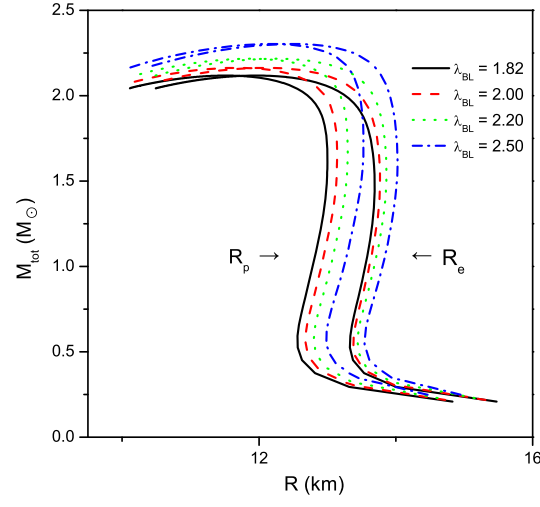

(a)

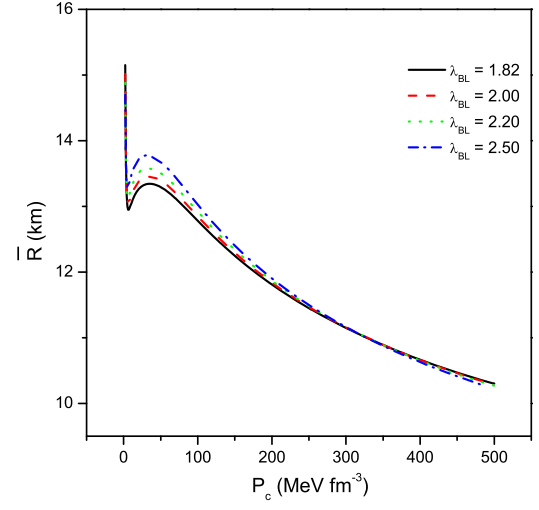

(b)

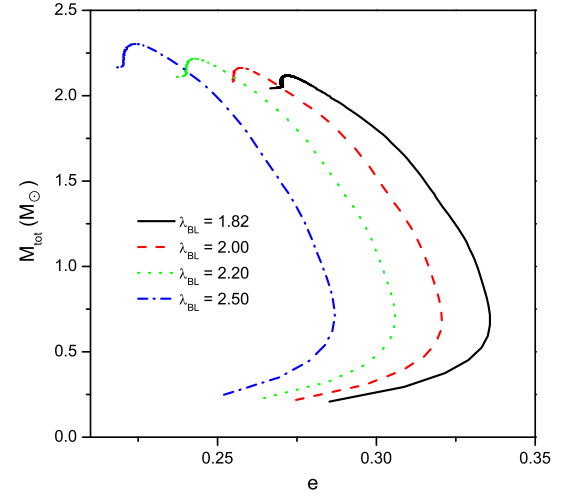

(c)
Fig. 3 Impact on anisotropic pressure on the star's radius. a The relation between radius and total mass. We label polar radius and equatorial radius as $R_{p}$ and $R_{e}$, respectively. The appearance of $R_{p}$ and $R_{e}$ shows that the star is deformed. The points where $R_{p}$ and $R_{e}$ overlap indicate no deformation at certain masses. $\mathbf{b}$ The relation between the pressure at the center and the average radius of the star. Here we can see that
NS's anisotropic pressure affects the obvious increment of NS's average radius, mainly in range $(5-400) \mathrm{MeV} \mathrm{fm}^{-3}$. c The relation between eccentricity and total mass. Star with a larger value of $\lambda_{B L}$ is harder to be deformed since curves of larger $\lambda_{B L}$ are closer to zero. Remember that while $e=0$, the object's shape is spherical becomes lower. This result is the consequence of Eqs. (7), (61), (62), and (72), as the increment of total mass is influenced by factors within those equations. Fig. $2 b$ shows mass correction for each value of $\lambda_{B L}$. The curves are plotted at certain pressure of center, i.e. $P_{c}=157 \mathrm{MeV} \mathrm{fm}^{-3}$, which is the point where star with $\lambda_{B L}=2.50$ reaches maximum mass. As we expect, the mass correction increment is not linear to $\lambda_{B L}$. This finding indicates that NS's anisotropic pressure has a significant impact on mass correction, which in the next turn affects the total mass, although the maximum value of mass correction we obtained only in the order of $10^{-2} M_{\odot}$. However, without HT formalism and any mass correction, the anisotropic pressure affects the star's mass increment. Please see the discussion o the mass-radius relation of isotropic NS in Ref. [3]. It means that in anisotropic NS, the mass can increase by increasing anisotropic contribution and rotation.

Another exciting result on the total mass that we have obtained is that the maximum mass for $\lambda_{B L}=2.50$ (i.e., the blue dot-dash curve) exceeds $2.25 M_{\odot}$. On the other hand, recently, LIGO/Virgo collaboration reported GW190814, with the secondary compact object has a mass of $2.6 M_{\odot}$ (Please see Ref. [17] for detailed discussions.). It is still unclear what the secondary compact object is, and it might be either a BH or an NS. Abbott et al. [17] argue that the secondary compact object is $\mathrm{BH}$. If it was a $\mathrm{BH}$, then it might be the lightest $\mathrm{BH}$ that has been observed. If it was an NS, it might be the heaviest NS that is ever observed, and the mass is significantly larger than the known maximum mass constraint. With the formalism we developed, we can obtain NS's maximum mass, reaching $2.6 M_{\odot}$. We are also optimistic that formalism might help a stable NS model with considerable mass. However, there are other details of EOSrelated things and anisotropic models of NS that we have not fully explored yet in this work, and this is already out of the scope of this work, and we leave it for our subsequent work.

Figure 3a shows the relation between radii, i.e., polar radius and equatorial radius, and total mass of the star. As a star rotates, deformation occurs. This behavior is like the deformation due to the rotation of the earth. The rotation causes the polar radius to be less than the equatorial radius. We labeled polar radius and equatorial radius as $R_{p}$ and $R_{e}$, respectively. Wen et al. [2] have worked on rotational deformation of isotropic NS, but there is an issue, i.e., the rotation is weak for slowly rotating NS. Other previous studies [21,32] showed that the deformation occurs at static anisotropic stars, but both also have issues that need to be understood. In Ref. [32], the authors reported no significant influence of varied anisotropic pressure on the star's deformation (see radius-mass relation and Ref. [32]). In this work, we obtained good results that handled those issues quite well. In our results, the variation of anisotropic strength impacts the star's shape. The deformations are not weak enough, which are similar compared to results in Ref. [21] (see radiusmass relation in Ref. [21]). In Fig. 4, we plotted 3- and 2dimensional illustration of NS in each particular pressure of the center for $\lambda_{B L}=1.82$. We can see that at lower mass, the star's shape tends to be more oblate. As the mass increase, the star is getting more spherical. This finding shows that the radius-mass relation becomes more compact as the mass increases. Note that at a certain mass for each curve, $R_{p}$ and $R_{e}$ overlap each other as we can see in Fig. 3a. Such a condition indicates that there is no pronounced deformation of the mass. 

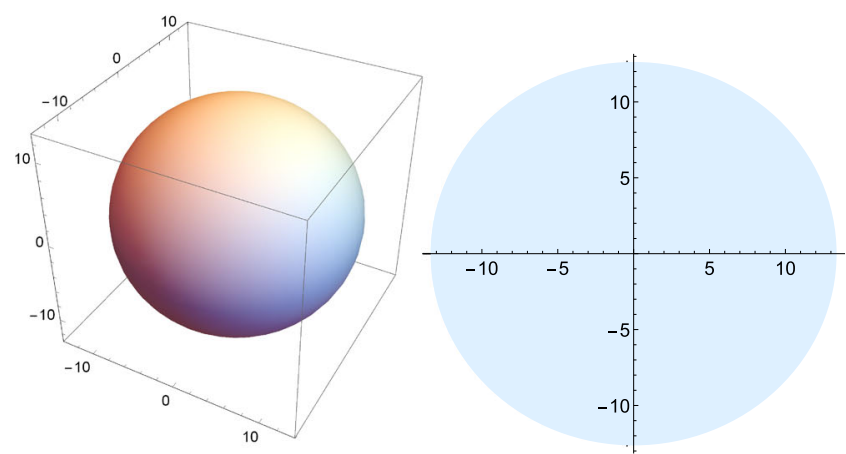

(a) $P_{c}=5 \mathrm{MeVfm}^{-3}$

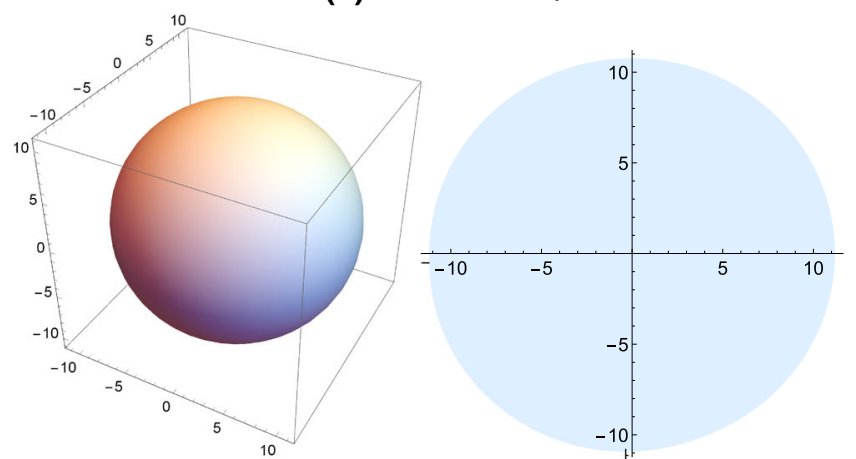

(c) $P_{c}=330 \mathrm{MeVfm}^{-3}$

Fig. 4 Illustration of 3- and 2-dimensional deformation of neutron stars in each particular pressure in center at $\lambda_{B L}=1.82$. Here we can see, stars with lower pressure in center are more oblate. At $P c=5 \mathrm{MeV} \mathrm{fm}-3, R_{p}=12.63 \mathrm{~km}$, and $R_{e}=13.37 \mathrm{~km}$; at

Figure $3 \mathrm{~b}$ shows the relation between the pressure at the center and the average radius of the anisotropic star. We define the average radius as

$\bar{R} \equiv \frac{R_{p}+R_{e}}{2}$

Generally, anisotropic pressure affects a star's average radius. The effect can be seen in pressure range about 5$250 \mathrm{MeV} \mathrm{fm}^{-3}$. The maximum average radius is different for each value of $\lambda_{B L}$, but it is clear that for every value of $\lambda_{B L}$ used, the average radius decreases when the pressure at the center exceeds $50 \mathrm{MeV} \mathrm{fm}^{-3}$. When the pressure at the center exceeds $250 \mathrm{MeV} \mathrm{fm}^{-3}$, the curves still decrease and tend to coincide until the pressure $=350 \mathrm{MeV} \mathrm{fm}^{-3}$, which indicates that anisotropic pressure does not significantly affect the average radius in this range. For the pressure range between 350 and $500 \mathrm{MeV} \mathrm{fm}^{-3}$ show that the curves are separated again.

Figure $3 \mathrm{c}$ shows the relation between eccentricity and total mass. We can see that larger eccentricity is associated with lower mass, so the star is easier to become more oblate at a lower mass. This fact is matched with what we can see in Figs. $3 \mathrm{a}$ and 4.

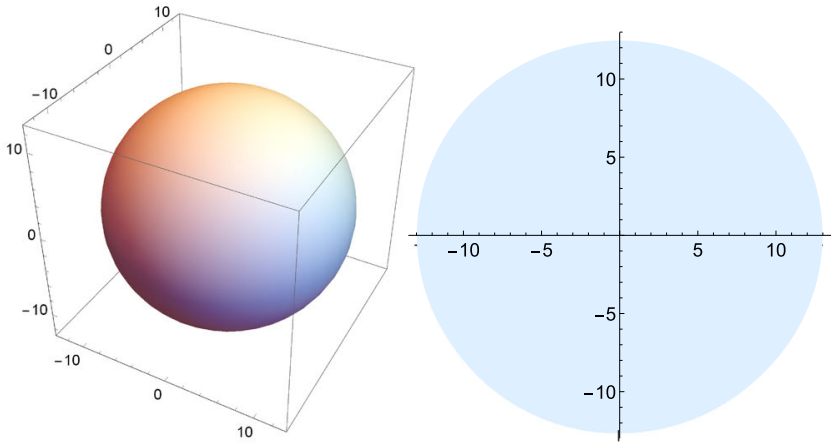

(b) $P_{c}=105 \mathrm{MeVfm}^{-3}$

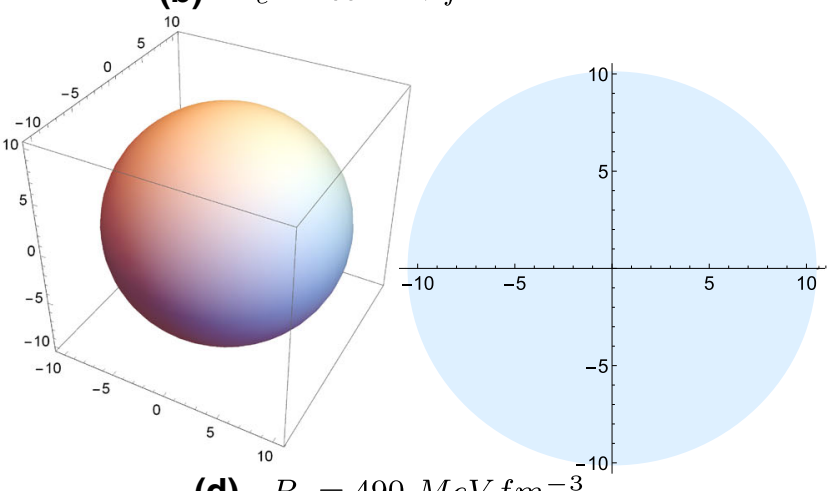

(d) $P_{c}=490 \mathrm{MeVfm}^{-3}$

$P c=105 \mathrm{MeV} \mathrm{fm} \mathrm{fm}^{-3}, R_{p}=12.46 \mathrm{~km}$, and $R_{e}=12.98 \mathrm{~km}$; $P c=330 \mathrm{MeV} \mathrm{fm} \mathrm{fm}^{-3}, R_{p}=10.78 \mathrm{~km}$, and $R_{e}=11.20 \mathrm{~km}$; and $P c=490 \mathrm{MeV} \mathrm{fm} \mathrm{fm}^{-3}, R_{p}=10.14 \mathrm{~km}$, and $R_{e}=10.52 \mathrm{~km}$

Another important insight we can get from Fig. $3 \mathrm{c}$ is the fact that stars whose stronger anisotropic contributions have less value of eccentricity since curves of larger $\lambda_{B L}$ are closer to zero. This condition is the same as the one obtained in Ref. [32], where the anisotropic pressure comes from a strong magnetic field of NS. However, in Ref. [32], the difference between each curve of eccentricity cannot be seen obviously (Please see radius-mass relation in Ref. [31].), which indicate different magnitudes of the magnetic field do not affect the eccentricity significantly. A highlight we can get from our result in Fig. $3 \mathrm{c}$ is the obvious effect of anisotropic pressure on the eccentricity. We can see that stars with weaker anisotropic terms tend to be easier to deform. From pressuremass relation in Fig. 2a and radius-mass relation in Fig. 3a, we can see that larger value of $\lambda_{B L}$ produces larger mass and smaller radius. This condition leads NS becomes harder to be deformed.

\section{Conclusion}

This work extends the analytical HT formalism for slowly rotating stars for isotropic matter by including the anisotropic 
pressure correction in the energy-momentum tensor. The results apply to the study of anisotropic NSs. To describe the EOS of NS matter, we use the RMF model with the BSP parameter set $[43,44]$ where the standard SU(6) prescription and hyperon potential depths [45] is used to determine the hyperon coupling constants. For the inner and outer crusts, we use the crust EOS obtained from Miyatsu et al. [46]. While for the anisotropic pressure model, we use the Bowers-Liang (BL) model for the anisotropic pressure of NS [42]. Note that the values of the anisotropic parameter of BL model $\lambda_{B L}$ can be interpreted as the strength of the anisotropic term. We have found that the impact of increasing $\lambda_{B L}$ value from $\lambda_{B L}=1.82$ to $\lambda_{B L}=2.50$ on angular velocity relative to local inertial frame at the surface, i.e. $\bar{\omega}(R)$ is the NS rotate faster. The corresponding increasing $\lambda_{B L}$ value also implies increment of mass and average radius of the NSs. It is also evident that NSs with larger mass are harder to be deformed.

Acknowledgements MLP sincerely acknowledges Indonesian Endowment Fund for Education (LPDP), Ministry of Finance, Republic of Indonesia for the fully funded scholarship. AS is partly supported by DRPM UI's (Skema PPI Q1 2021; PUTI-Q1 and PUTI-Q2) Grants No: ST-811/UN2.F3.D/PPM.00/2021; NKB-1368/UN2.RST/ HKP.05.00/2020; NKB-1647/UN2.RST /HKP.05.00/ 2020.

Data Availability Statement This manuscript has no associated data or the data will not be deposited. [Authors' comment: This is a theoretical work. Therefore, it is not necessary to deposit the data. If anybody wants our code and the data from our calculation results, he/she can simply send a message to one of our email addresses.]

Open Access This article is licensed under a Creative Commons Attribution 4.0 International License, which permits use, sharing, adaptation, distribution and reproduction in any medium or format, as long as you give appropriate credit to the original author(s) and the source, provide a link to the Creative Commons licence, and indicate if changes were made. The images or other third party material in this article are included in the article's Creative Commons licence, unless indicated otherwise in a credit line to the material. If material is not included in the article's Creative Commons licence and your intended use is not permitted by statutory regulation or exceeds the permitted use, you will need to obtain permission directly from the copyright holder. To view a copy of this licence, visit http://creativecomm ons.org/licenses/by/4.0/.

Funded by SCOAP ${ }^{3}$.

\section{References}

1. I. Vidaña, Eur. Phys. J. Plus 133, 445 (2018)

2. D.H. Wen, W. Chen, L.G. Liu, Chin. Phys. Lett. 22, 1604 (2005)

3. A. Rahmansyah, A. Sulaksono, A.B. Wahidin, A.M. Setiawan, Eur. Phys. J. C 80, 769 (2020)

4. N.K. Glendenning, Compact Stars: Nuclear Physics, Particle Physics, and General Relativity (Springer, New York, 1997)
5. P.B. Demorest, T. Pennucci, S.M. Ransom, M.S.E. Roberts, J.W.T. Hessels, Nature 467, 1081 (2010)

6. E. Fonseca et al., Astrophys. J 832, 167 (2016)

7. see also related recent report in Z. Arzoumanian et al., Astrophys. J. Suppl. Ser. 235, 37 (2018)

8. H.T. Cromartie et al., Nat. Astron. 4, 72 (2020)

9. J. Antoniadis et al., Science 340, 1233232 (2013)

10. F. Özel, P. Freire, Ann. Rev. Astron. Astrophys. 54, 401 (2016)

11. S. Guillot et al., Astrophys. J. Lett. 887, 27 (2019)

12. S. Bogdanov et al., Astrophys. J. Lett. 887, 25 (2019)

13. S. Bogdanov et al., Astrophys. J. Lett. 887, 26 (2019)

14. B.P. Abbott et al., Phys. Rev. Lett. 119, 161101 (2017)

15. B.P. Abbott et al., Phys. Rev. Lett. 121, 161101 (2018)

16. B.P. Abbott et al., Phys. Rev. X 9, 011001 (2019)

17. R. Abbott et al., Astrophys. J. 896, L44 (2020)

18. P. Haensel, M. Fortin, J. Phys. Conf. Ser. 467, 012034 (2017)

19. M. Dutra et al., Phys. Rev. C 90, 055203 (2014)

20. O. Lourenço, M. Dutra, C.H. Lenzi, C.V. Flores, D.P. Menezes, Phys. Rev. C 99, 045202 (2019)

21. R. Mallick, S. Schramm, Phys. Rev. C 84, 012034 (2014)

22. B.A. Li, P.G. Krastev, D.H. Wen, N.B. Zhang, Eur. Phys. J. A 55, 117 (2019)

23. Y. Lim, J.W. Holt, Eur. Phys. J. A 55, 209 (2019)

24. J. Oppenheimer, G.M. Volkoff, Phys. Rev. 55, 374 (1939)

25. J.B. Hartle, Astrophys. J. 150, 1005 (1967)

26. J.B. Hartle, K.S. Thorne, Astrophys. J. 153, 807 (1968)

27. E. Berti, F. White, A. Maniopoulou, M. Bruni, Mon. Not. R. Astron. Soc. 358, 923-938 (2005)

28. V.S. Manko, E.W. Mielke, J.D. Sanabria-Gómez, Phys. Rev. D 61, 081501(R) (2000)

29. G.B. Cook, S.L. Shapiro, S.A. Teukolsky, Astrophys. J. 424, 823 (1994)

30. A. Sulaksono, Int. J. Mod. Phys. E 24, 1550007 (2015)

31. R. Rizaldy, A. Sulaksono, J. Phys. Conf. Ser. 1011, 012083 (2018)

32. R. Rizaldy, A. Sulaksono, J. Phys. Conf. Ser. 1080, 012031 (2018)

33. H.O. Silva, C.F.B. Macedo, E. Berti, L.C.B. Crispino, Class. Quantum Gravity 32, 145008 (2015)

34. I. Lopes, G. Panotopoulos, A. Rincón, Eur. Phys. J. Plus 134, 454 (2019)

35. M.D. Danarianto, A. Sulaksono, Phys. Rev. D 100, 064042 (2019)

36. A. Wojnar, H. Velten, Eur. Phys. J. C 76, 697 (2016)

37. J. Ovalle, Phys. Rev. D 95, 104019 (2017)

38. V.I. Afonso, G.J. Olmo, D. Rubiera-Garcia, Phys. Rev. D 97, $021503(2017)$

39. V.I. Afonso, G.J. Olmo, E. Orazi, D. Rubiera-Garcia, Eur. Phys. J. C 78, 866 (2018)

40. K. Konno, T. Obata, Y. Kojima, Astron. Astrophys. 352, 211-216 (1999)

41. C. Sivaram, K. Arun, Open Astron. J. 5, 7-11 (2012)

42. R.L. Bowers, E.P.T. Liang, Astrophys. J. 88, 657 (1974)

43. B.K. Agrawal, A. Sulaksono, P.G. Reinhard, Nucl. Phys. A 882, 1 (2012)

44. A. Sulaksono, B.K. Agrawal, Nucl. Phys. A 895, 44-58 (2012)

45. J. Schaffner-Bielich, A. Gal, Phys. Rev. C 62, 034311 (2000)

46. T. Miyatsu, S. Yammamuro, K. Nakazaki, Astrophys. J. 777, 4 (2013) 